\title{
AGENTE, SUBJETIVACIÓN Y EDUCACIÓN CORPORAL:
}

\section{REFLEXIONES METODOLÓGICAS}

\author{
LIC. MARÍA CAROLINA ESCUDERO \\ Licenciada en Sociología por la Universidad Nacional de La Plata. Ayudante Diplomada en la \\ Licenciatura em Trabajo Social de la Universidad Nacional de La Plata. Investigadora del Grupo de \\ Estudios en Educación Corporal del Centro de Interdisciplinario de Metodología de las Ciencias \\ Sociales. Becaria del Consejo Nacional de Investigaciones Científicas y Tecnológicas \\ - CONICET (La Plata - Buenos Aires - Argentina) \\ e-mail: carolinaescu@yahoo.com.ar
}

\begin{abstract}
RESUMEN
El objetivo del trabajo se orienta a presentar algunas consecuencias metodológicas resultantes de adoptar una postura epistemológica foucaulteana para investigar al sujeto, al cuerpo y sus posibles relaciones con la educación corporal. Para ello, trabajaré con la concepción de agente que propone Bourdieu y con la idea de modo de subjetivación que presenta Foucault, indicaré diferencias de acepción y de sentido. Luego vincularé estas diferencias con lo que entiendo son dos modos básicos de comprender el cuerpo; como medio de individuación e instancia de objetivación del sujeto o como modo de subjetivación y singularización. Por último explicitaré las implicaciones que estas diferentes posiciones epistemológicas presentan para una práctica de trasmisión de saber en educación corporal.
\end{abstract}

PALABRAS CLAVE: Educación corporal; sujeto; cuerpo; subjetivación. 


\section{PRESENTACIÓN}

El objetivo de este trabajo es presentar lo que considero implicaciones metodológicas diferenciales respecto de la enseñanza en educación corporal. Estas diferencias se sostienen en las diversas consecuencias prácticas que para el acto de trasmisión de saber tiene concebir al otro de la relación, en términos de agente o en términos de un sujeto.

El interés que motiva estas reflexiones encuentra su sostén en la tarea de investigación que vengo desarrollando con el Grupo de Investigación sobre Metodología y Educación corporal que coordina Ricardo Crisorio en la UNLP. El mismo se inscribe en una línea de análisis que apunta a introducir una teoría de lo simbólico en el campo de las investigaciones sobre Educación Física. Esto genera como primer consecuencia un desplazamiento desde aquel concepto hacia el de Educación Corporal y refuerza una segunda característica de los intereses del grupo; el sesgo metodológico e interdisciplinar al que apunta, como clave interpretativa y heurística para producir saber sobre el cuerpo y sobre la educación corporal. Así, el campo de estudios en que se desarrolla el presenta artículo cruza intereses de la Educación Física/Educación Corporal, con desarrollos teóricos de las Ciencias Sociales y del Psicoanálisis. En este sentido, estas reflexiones presentan un carácter parcial respecto de la historia investigativa del grupo y del horizonte actual de investigación, sin embargo es deudora en su interés, de los debates y líneas de análisis que en él se desarrollan.

El trabajo tendrá tres partes; en la primera explicitaré lo que entiendo y de qué manera utilizo la expresión educación corporal, en la segunda desarrollaré los conceptos de agente y subjetivación, utilizando exclusivamente referencias a Bourdieu y Foucault. Explicitaré de manera sintética y esquemática las relaciones que tales categorías proponen en relación al cuerpo y por último, presentaré las reflexiones o inquietudes surgidas en relación a las diversas implicancias metodológicas respecto de la educación corporal que aquéllas diferencias conllevan.

Voy a presentar qué se entiende y cómo se utiliza el término educación corporal por parte del Grupo de estudios en Educación Corporal (GEEC) que coordinan Ricardo Crisorio y Marcelo Giles ${ }^{1}$. En primer lugar mencionaré, que

I. Grupo de Estudios en Educación Corporal, es un grupo de investigación que trabaja en la Facultad de Humanidades y Ciencias de la Educación de la Universidad Nacional de La Plata, forman parte 
dentro del equipo de investigación, el término se presenta con un interés político, el de oponerlo a educación física. Sin embargo, si se observa el potencial significante de la expresión es dable reconocer allí la posibilidad de pensar en los términos de la educación corporal la enseñanza de toda una serie de prácticas que implican al cuerpo y que trascienden a la propia educación física. De hecho quien escribe no proviene del campo de la educación física y sin embargo, se identifica con el interés puesto en juego al hacer circular en el ámbito institucional de las humanidades, la expresión educación corporal. Especialmente si se tiene en cuenta que la carrera de Educación Física en el contexto institucional de la Facultad de Humanidades y Ciencias de la Educación, ha sido conceptuada la mayoría de las veces como una disciplina eminentemente práctica -queriendo significar con esto, que está alejada de la teoría, del pensamiento y de la investigación-. Los intereses puestos en movimiento con la categoría educación corporal, tocan lugares comunes de diversas disciplinas entonces; la propia educación física, las ciencias de la educación y la sociología.

¿Por qué tiene un sentido diferente esta expresión? ¿Qué pone en cuestión su uso? Busca complejizar una serie de miradas y teorías que tienden a concebir el entrenamiento técnico, competente, del cuerpo con operaciones que se orientan exclusivamente a la physis, al sustrato biológico y orgánico de la persona. Esto implica algunas cuestiones; romper con la identificación cuerpo/organismo y mostrar que la mediación técnica y competente con el mundo no tiene una connotación negativa per se. También pone en cuestión la idea misma de educación, si por ello se entiende la traducción a una situación determinada de un conocimiento supuesto universal que tiene por objeto la incorporación a ese universal del mayor número de casos posibles.

Ahora bien, ¿Por qué no hablar sencillamente de educación? ¿O de formas de mediación del hombre con el mundo? No sólo porque el ámbito de formación, reflexión y construcción de conocimiento que lleva adelante el equipo de investigación es el de la educación corporal, sino porque hablar de cuerpo puede pensarse como una excusa para hablar del "problema del sujeto". Decir cuerpo es un modo de decir sujeto y es en este punto dónde la educación corporal hace su apuesta más fuerte. No podemos poner en cuestión la idea de entrenamiento técnico o la idea de educación si no pensamos en relación a quien lo hacemos. En este sentido, la educación corporal se orienta a criticar una figura del hombre y a construir si no

del mismo, un importante número de investigadores formados en Educación Física, investigadores en formación y becarios. Así, mismo articula el trabajo de investigación con la docencia en grado y en posgrado. El grupo ha organizado dos coloquios sobre cuerpo y ha participado en la formación de una mesa sobre cuerpo en las Jornadas de Sociología de la UNLP. 
una nueva figura, si unos procedimientos diferenciales que puedan utilizarse en los procesos de encuentro de un sujeto con otro. Procesos que, en la medida que abandonan las orientaciones universalistas o totalizantes, naturalistas u organicistas, dan por resultado la formación de encuentros de enseñanza ligados a la lógica de la transferencia y orientados a producir modos de subjetivación particulares. Así, Giles sostiene

Si pretendemos cambiar las prácticas para dominar los saberes y haceres que posibiliten la reflexión en sus distintos ámbitos, debemos comenzar a pensar en términos de una educación corporal, entendiendo que el cuerpo es más que organismo y que los saberes que trasmitimos construyen un saber práctico, público (GILES, 2007. p. 5).

Quisiera sintetizar ahora, la idea de cuerpo que subyace a la apuesta de la educación corporal. Podría decir: "hay cuerpo porque hay lenguaje"; sin embrago esto no deja de ser una frase hecha que amerita al menos algunas explicitaciones de uso. No quiere decir simplemente que el lenguaje deja una marca en términos de inscripción de la cultura en el organismo, esto acota demasiado el problema del cuerpo y si bien no lo reduce a una asimilación-acomodación con el medio en términos orgánicos, sí supone cierta reducción del sujeto al medio "cultura". Tampoco quiere decir que hay una experiencia originaria y primera del cuerpo que se pueda traducir en palabras de manera completa: porque lo decimos existe a modo de un hiperconstructivismo lingüístico. Acá quisiera evitar interpretaciones que apunten a una doble reducción: tanto una reducción organicista que sostenga que hay un resto, animalidad o pulsión en lo humano que se sostienen como tal y que ahí habría que buscar el cuerpo, como una reducción culturalista que suponga que todo que hay de cuerpo puede traducirse de manera transparente y sin equívocos al lenguaje o a lo simbólico.

Que el lenguaje haga aparecer al cuerpo, no quiere decir que podamos capturar al cuerpo con el lenguaje, el lenguaje siempre se distancia de la intención y la voluntad. En esa distancia es en la que hay que ubicarse para evitar reduccionismos y capturar lo particularidad de la experiencia del cuerpo, o quizás debería decir, de la experiencia de subjetivación por el cuerpo.

En este sentido, puedo decir que el lugar del cuerpo es un poco más difícil de circunscribir y de enunciar, lo mismo que el lugar del sujeto que lo construye y lo habla. El lenguaje como tal funciona como divisor, como espacio lógico a partir del cual un organismo deviene cuerpo y en tanto espacio lógico cumple una función que no es la de traducir, explicar o nombrar sin equívocos las experiencias subjetivas, 
el sujeto no puede usar el lenguaje como le de la gana. Funciona, en este sentido, para abrir un espacio en el que situar a un otro; sólo así se vuelve posible pensar el cuerpo y el sujeto como construcciones simbólicas no reductibles al medio cultural. No hay nada de natural en el cuerpo y en el sujeto porque no hay nada de natural en la forma en que el sujeto y el cuerpo se encuentran con el mundo. Tampoco hay nada de natural en la relación del sujeto con su cuerpo porque allí también el encuentro está mediado simbólicamente y si bien muchas veces los efectos de subjetivación se localizan en el organismo biológico, hay allí un sujeto que interpreta esos efectos y les asigna sentido. El cuerpo y el sujeto que suponen la educación corporal no están pre hechos ni terminados, devienen en la relación con un otro.

Citemos un texto de Nancy para apoyar esta posición:

\begin{abstract}
De ahí que no sea posible escribir "al" cuerpo, o escribir "el" cuerpo, sin rupturas, cambios de parecer, discontinuidades (discreción), ni tampoco sin inconsecuencias, contradicciones, desviaciones dentro del discurso mismo. Hace falta meterse por entre este "sujeto" y este "asunto", al que la palabra cuerpo, por sí sola impone una dureza seca, nerviosa, que hace restallar las frases donde se la emplea.
\end{abstract}

Quizás cuerpo es la palabra sin empleo por antonomasia. Quizás es, de todo lenguaje, la palabra de más. (NANCY, 2003, p 19)

Voy a acotar el análisis de la categoría de agente, al sentido que le asigna Bourdieu (1996). Para ello voy a utilizar distintos textos de referencia. En cuanto a la noción de subjetivación, voy a referirme a Foucault: tanto a sus cursos, como a sus libros y diversas entrevistas (FOUCAULT, 1999, 2002, 2003b, 2003b). Vale aquí una aclaración: en el parágrafo anterior utilicé la expresión sujeto, categoría que Foucault no utiliza en los términos desarrollados. El equivalente en Foucault a lo que en el apartado anterior fue nombrado con la palabra sujeto viene dado por la palabra subjetivación (FOUCAULT, 2002, 2003b).

Respecto del trabajo con la bibliografía quisiera hacer una aclaración, que puede servir simplemente como excusa o puede interpretarse como recurso de método para avanzar y sistematizar algunas reflexiones. En este segundo sentido me gustaría que se entienda: si bien los autores trabajados pueden ser matizados de diversos modos, ya sea en el contexto de la totalidad de su producción teórica como el caso de Bourdieu por ejemplo y mostrar el esfuerzo que el autor ha 
hecho sistemáticamente para dar lugar a la producción vía la acción (BOURDIEU, 1996, 2000). O también puede relativizarse la lectura "positiva" respecto de la categoría de gobierno de sí foucaulteana e inscribir la emergencia de esta categoría en la totalidad del desarrollo del pensamiento de Foucault para mostrar cómo, otrora se ocupó más de las formas de reproducción y disciplinamiento que de formas de producción de subjetivación novedosas. Creo sin embrago que haberme detenido es esos matices, hubiera significado posponer un cierto cierre -parcial- que presento en este texto. La idea, el interés genuino es investigar en el largo plazo sobre estos elementos y si bien la instancia de escritura pareciera sentenciar una posición, el movimiento del pensamiento imprime vitalidad o nos permite revisar esa sentencia. Además, el recurso interpretativo a extremar posiciones ayuda a mostrar y hacer visible lo que se quiere decir, cuestión que muchas veces, por falta de vocabulario preciso o por el carácter embrionario de la idea resiste en hacerse ver.

En este sentido el recurso a la cita es un recurso cuasi-técnico, de manera general podría decir que no operan como cita de autoridad, sino como ejemplos específicos y acotados que representan, en el contexto de mis reflexiones, una visualización o concepción del sujeto/agente y del cuerpo sobre la cual tendría sentido o no, situarse. En este artículo, la idea no es presentar una reflexión sobre los autores, sino usar a los autores como herramientas para pensar un problema o campo problemático: el de la educación corporal y la producción de subjetividad.

Respecto de Bourdieu, me gustaría destacar que aunque su esfuerzo está orientado a superar algunas dicotomías clásicas del pensamiento social (BOURDIEU, 1996); sin embargo, su noción de agente no alcanza para resolver el problema. Es imposible dejar de referir la noción de agente a la relación que el mismo establece con el habitus, la illusio, el sentido práctico y las estrategias, todo lo cual está orientado a brindar una visión de la acción social que no se reduzca a la aplicación autómata/ automática de una regla social/estructural (BOURDIEU, 2000). Sin embargo, y más allá de los esfuerzos de Bourdieu por brindar definiciones del agente que incorporen un margen de libertad de acción, un cierto nivel de producción de realidad y de generación de principios de organización del mundo, creo que finalmente termina por rellenar ese espacio y ese margen con un "determinismo" socio/culturalista que reenvía la noción de agente a la incorporación histórica y relacional de estructuras materiales y simbólicas existentes. Si bien tiene en cuenta y teoriza el lugar del otro en la configuración del agente, creo que no entiende esa función en términos de una apertura y diferencia que impliquen procesos de desidentificación y, en ese sentido, de "construcción de subjetividad" - volverse sujeto -. Por el contrario el lugar del otro en la configuración del agente se reduce a asignarle una identidad, a 
brindarle criterios de identificación, nombrarlo y asignarle una posición en el espacio simbólico (BOURDIEU, 1996).

Termina, en un sentido fuerte, volcado al reproductivismo y la explicación de su dinámica de funcionamiento; no hay un desarrollo explicativo semejante del lugar del agente en instancias de resistencia que configuren nuevos órdenes o reglas de campo. Se puede ver la dimensión simbólico-política de la dominación pero no la dimensión simbólico-política en la constitución de los agentes, más allá del lugar de resistencia dado por la posición y trayectoria en el campo.

Creo que esa ausencia habla de cierto límite en la construcción de su categoría, lo que se podría explicar en base al interlocutor con quien discute, no sólo el estructuralismo sino también las teorías de la elección racional y allí, por mostrar que no todo es cálculo racional en la lógica de los intereses y las estrategias, introduce la dimensión simbólica pero para reducir a ella la explicación del agente sin situar allí una relación compleja, de apertura, división, incompletud y encuentro particular.

En algunos ejemplos en los que refiere al cuerpo puede verse esto claramente:

El mundo es comprensible, inmediatamente dotado de sentido, porque el cuerpo, gracias a sus sentidos y a su cerebro, tiene la capacidad de estar presente en el exterior de sí mismo, en el mundo, y de ser impresionado y duraderamente modificado por él, habiendo estado largamente desde el origen expuesto a sus regularidades. Al haber adquirido por ello un sistema de disposiciones acorde a tales regularidades, resulta inclinado y apto para anticiparlas prácticamente en conductas que comprometen un conocimiento por el cuerpo que garantiza una comprensión del mundo totalmente diferente del acto intencional de desciframiento consciente que por lo general se pone bajo la idea de comprensión (CHAUVIRÉ; FONTAINE, 2008, p. 34).

Y también:

Las propiedades corporales, en tanto que productos sociales, son aprehendidas a través de categorías de percepción y de sistemas sociales de clasificación que no son independientes de la distribución de las diferentes propiedades entre las clases sociales: las taxonomías al uso tienden a oponer, jerarquizándolas, las propiedades más frecuentes entre los que dominan (es decir, las más raras) y las más frecuentes entre los dominados. La representación social del cuerpo propio, con la que cada agente social ha de contar desde que nace para elaborar la representación subjetiva de su cuerpo (y más soterradamente, su hexis corporal), es pues el resultado de la aplicación de un sistema de clasificación social cuyo principio regulador es el mismo que el de los productos sociales a los que se aplica (BOURDIEU, | 986, p. |85-|86). 
Veamos ahora a Foucault. Si bien es preciso reconocer la importancia que en los análisis de Foucault tiene la constitución del sujeto en términos de objetivación de saberes, prácticas y discursos, es oportuno indicar el cambio de perspectiva que opera a partir de Hermenéutica del sujeto (2002) y de Historia de la Sexualidad (2003). Aparece la idea de modos de subjetivación ligados a prácticas de constitución de sí, dónde la categoría de gobierno no se vincula ni al poder soberano sobre la muerte, ni a la gestión de la vida sino a la constitución de una ética que puede pensarse como una estética de la existencia ${ }^{2}$. Aquí está la clave de lectura que propongo para salirnos de la interpretación hegemónica sobre Foucault, sesgada hacia Vigilar y Castigar (1989). Debemos vincular a esto otro cambio que habla de la posibilidad misma de resistencia. Al principio Foucault hablaba de transgresión y pensaba su posibilidad como un afuera del dispositivo; con la idea de resistencia esto cambia y empieza a entenderse como posible sólo si es inmanente al mismo. Es preciso haber sido objeto de otro y también objeto de sí para poder volverse sujeto y además, es preciso aprender a regular la propia estética en una ética. Hay un saber hacer que implica un aprendizaje y como tal, un método (FOUCAULT, 2003a, 2003b).

Esto nos remite a la pregunta por cómo el sujeto se gobierna a sí mismo, qué prácticas establece como modos de ser en la relación que entabla consigo mismo, qué pragmática de la vida en el ejercicio positivo del poder hacer instituyen ciertas prácticas de subjetivación que se acompañan de instancias de decisión y libertad. El ejercicio positivo del poder, la constitución de sí como sujeto ético implica una lógica del aprendizaje, uno tiene que cultivarse en modos de sujeción que son propios y alternativos a los modos de sujeción del poder del otro y estas prácticas de sí, este buen gobierno de sí comienzan a materializarse en relación al cuerpo. El cuerpo entendido como el objeto a partir del cual es posible subjetivarse en base a prácticas determinadas y particulares, donde el concepto de práctica implica una actitud y un modo de vida que se articulan más con la lógica del resultado que con la de la intención (FOUCAULT, 2002, 2003a, 2003b).

2. De los tres momentos en los que se ha caracterizado la producción foucaulteana y sin ánimo de apoyar taxativamente esta caracterización; la arqueología del saber, la genealogía del poder y la genealogía de la moral (o período ético), podría decir que me apoyo en lo desarrollado por el autor en la última etapa, que coincide en términos cronológicos, con las reflexiones que lleva adelante sobre el problema del gobierno en general. Cabe destacar en este sentido que la problemática del gobierno en Foucault no se reduce a prácticas de acceso del sujeto a la verdad (en el sentido de la parresia cínica), implica también otras formas ligadas al conocimiento de sí, la dirección de conciencias y las formas confesionales de la pastoral cristiana. A los efectos de estas reflexiones, considero la primer acepción. 
El vínculo entre gobierno de sí, ética y estética, implica poder hacer de la propia vida una obra en constante creación, crearla no de una vez y para siempre sino permanentemente.

Veamos qué dice Foucault;

Con el cristianismo se produjo un lento, gradual desplazamiento en relación a la moralidad de la antigüedad, que era esencialmente una práctica, un estilo de libertad. Por supuesto también ha habido ciertas normas de conducta, que gobernaban el comportamiento de cada individuo. Pero el deseo de ser un sujeto de moral, y la búsqueda de una ética de la existencia, eran en la antigüedad solamente un intento de afirmar la propia libertad y dar a la propia vida una cierta forma (...) Esta elaboración de la propia vida como una obra de arte personal si bien obedecía a ciertos cánones colectivos, estaba en el centro, pienso, de la experiencia moral, la voluntad de moralidad en la antigüedad; mientras en el cristianismo, con el texto religioso la idea de voluntad de dios, el principio de la obediencia, la moralidad tomó gradualmente la forma de un código de normas (FOUCAULT apud KAMINSKY, 2003, p. 134).

Y también,

En primer lugar, yo realmente creo que no existe un sujeto soberano, fundacional, una forma universal de sujeto que pueda hallarse en cualquier parte (...) Creo, por el contrario, que el sujeto es constituido a través de prácticas de sujeción, o de una manera más autónoma, a través de prácticas de liberación, de libertad, como en la antigüedad; sobre la base, por supuesto, de una cantidad de reglas, estilos, invenciones que se pueden encontrar en el entorno cultural (FOUCAULT apud KAMINSKY, 2003, p. 136).

III

Una conclusión parcial respecto de Foucault y Bourdieu³: o se tiene un cuerpo o se es un cuerpo. O se es un agente determinado y capaz de agencia o se practican modos de sujeción o subjetivación que lo vuelven a uno sujeto. Acá encontramos una diferencia de cualidad respecto a cómo entender al cuerpo y el sujeto y por tanto a cómo entender las implicaciones metodológicas que tales concepciones tienen para una educación corporal.

Ahora voy a presentar alguna reflexiones que aparecen como indicios para pensar otros modos de educación, las que están orientadas a sentar

3. Parcial en el sentido en que se sitúa en las diferencias entre ambos autores. Queda llevar adelante un proceso de reflexión que busque en cada uno de los autores puntos de contacto, de diálogo. Para luego usar ese lugar común como excusa del pensamiento, como herramienta con la que trabajar sobre el sujeto, el cuerpo y la educación corporal. 
posición y a explicitar un punto de partida posible entre otros; el elegido por quien escribe, para articular sus prácticas corporales a sus modos de generar y trasmitir saber.

Habíamos dicho que la educación corporal es una manera de poner sobre el tapete la pregunta por el sujeto, y hacerlo supone también poner en cuestión qué se entiende por educación. Aquí dos cuestiones que tienen que ver con las posiciones extremas que marqué hace unos instantes: se es un cuerpo o se tiene un cuerpo. Si se es un cuerpo, nada se puede aprehender de él más que estar atento a su devenir.

Colocar al cuerpo en el lugar del ser lo naturaliza y, por más intentos de historizar la forma cuerpo, y de situar en él las impresiones que la historia y lo social le cargan, esto no hace más que complejizar la visión naturalista que reduce el cuerpo al funcionamiento de un organismo en un medio, sólo que ahora el medio tiene historia y es producto humano, lo que en nada cambia, en sentido estricto, el lugar del cuerpo en la pregunta por el sujeto y menos aún en la pregunta acerca de cómo educar al sujeto en prácticas corporales. Cuando el cuerpo ocupa el lugar del ser, tiende a reducirse a lastre, muestra lo que no puede ser de otro modo y carga con el peso de la prueba por la materialidad que reviste. En esta perspectiva pierde sentido mismo la educación, ¿qué se le puede enseñar a algo que es lo que de manera inmediata me pone en relación con el mundo? Lo que redunda en que no habría necesidad de mediación simbólica, porque lo simbólico ocupa el lugar del mundo y no el lugar lógico de entre, de mediación o de acto del sujeto significando. ¿Qué puede aprender aquello que representa el ser del agente en el mundo social? ¿Qué potencial de ser otra cosa que mera representación y superficie de mostración inmediata de lo que tocó en suerte? Quizás esté extremando las preguntas y esté extremando el ser un cuerpo, pero esto permite visualizar el problema.

Del otro lado iqué pasa cuando el cuerpo es un objeto? ¿Cuándo el cuerpo se tiene y se usa? Se lo puede modelar, entrenar, educar, para bien o para mal, no me interesan en este punto las valoraciones morales de una buena o mala educación. Sí me interesa indicar que sólo el estatuto de objeto en cuanto tal -tanto del cuerpo como del sujeto- es lo que abre la posibilidad de pensar un otro/ Otro significante y en ese sentido operar una desnaturalización del sujeto. Esto además en la medida en que un mismo objeto, el cuerpo, no significa lo mismo para quien lo porta que para quien lo educa y es esa distancia de sentido aparece la capacidad de escucha y diálogo, apertura de una diferencia en la cual situar el acto educativo como acto de construcción. El cuerpo no es lo que tiene que ser. Es lo que puede hacerse de él y lo que se puede hacer con lo que hicieron 
del sujeto y del cuerpo. El cuerpo no representa una posición y una trayectoria social, el cuerpo es construcción de sentido y en su materialidad (organismo biológico) vemos los efectos/excesos de sentido que restan significarse. Por eso es un objeto en constante construcción, porque siempre hay exceso de sentido y siempre hay otro significante (sujeto o cuerpo). Aquí el acto de educar adquiere una importancia fundamental.

Quisiera marcar un límite: resulta posible en espacios acotados ya que no deja de ser una lógica de la práctica que escapa a la masificación (aunque no a la generalización). En ese sentido es preciso abrir la pregunta acerca de las condiciones de la educación actual para pensar modos de vehiculizar una apuesta política como ésta.

\section{Agent, subjection and corporal education: methodological reflections}

ABSTRACT: The aim of this work is to present some methodological implications resulting from adopting a Foucauldian epistemological position to investigate the subject, the body and its possible links with corporal education. To do this I explain the conception of subject and indicate differences aforementioned meaning and use in respect of the proposed Bourdie's category of agent. Then force between these differences with what I understand are two basic ways of understanding the body; as a means of individuation and instance of objectification of the subject or as a way of subjection and singling. Finally I explain the implications these have different epistemological position constraint for a practice of transmitting knowledge in corporal education.

KEY WORDS: Corporal education; subject; body; subjection.

\section{Agente, sujeição e educação corporal: reflexões metodológicas}

RESUMO: O objetivo do presente trabalho é apresentar algumas consequências metodológicas resultantes da adoção de uma postura epistemológica foucaultiana para pesquisar o sujeito, o corpo e suas possíveis relações com a educação corporal. Para tanto, trabalho com a concepção de agente proposta por Bourdieu e com a noção de modo de subjetivação, apresentada por Foucault, indicando diferenças de concepção e sentido entre elas. Logo após, vinculo essas diferenças com aquilo que entendo ser duas maneiras básicas de compreensão do corpo: como meio de individuação e instância de objetivação do sujeito, como modo de subjetivação e singularização. Por último, explicito as implicações que essas diferentes posições epistemológicas apresentam para uma prática de transmisão de saberes na educação corporal.

PALAVRAS-CHAVE: Educação corporal; sujeito; corpo; sujeição. 


\section{REFERENCIAS}

BOURDIEU, P. Notas provisionales sobre la percepción social del cuerpo. In: WRIGHT MILLS,

C. et al. Materiales de sociología crítica. Madrid: La piqueta, 1986. p. I83-194. . Cosas dichas. Barcelona: Gedisa, 1996. . Sentido práctico. España: Taurus, 2000.

CHAUVIRÉ, CH.; FONTAINE, O. El vocabulario de Bourdieu. Buenos Aires: Atuel, 2008. FOUCAULT, M. Estética, ética y hermenéutica. Barcelona: Paidós, 1999. (Obras esenciales. Volumen III) . Hermenéutica del sujeto. Argentina: Fondo de Cultura Económica. 2002. Historia de la sexualidad. Tomo II. El uso de los placeres. Argentina: Siglo XXI, 2003a.

$X X I, 2003 b$.

. Historia de la sexualidad. Tomo III. La inquietud de sí. Argentina: Siglo

GILES, M. Educación física y educación corporal: matrices corporales. In: ENCUENTRO DE CUERPO Y CULTURA DE LA UNLP, I. La Plata: Mecanografiado, 2007.

KAMINSKY, G. (Org.). El yo minimalista y otras conversaciones con Michel Foucault. Buenos Aires: La Marca-Biblioteca de la mirada, 2003. (Entrevistas)

NANCY, J. L. Corpus. Madrid, Arena Libros. 2003.

Recebido: 14 abr. 2010 Aprovado: 05 jan. 2011

Dirección para correspondencia:

María Carolina Escudero Calle $469 n^{\circ} 1246$ entre calles 7 y 7 bis. Barrio Savoia - City Bell - C.P 1896. La Plata - Argentina 